Matos-Duarte, M.; Berlanga, L.A. (2020) Effects of Exercise on Flexibility in Adults over 65 Years and Older. Revista Internacional de Medicina y Ciencias de la Actividad Física y el Deporte vol. 20 (80) pp. 611-622 Http://cdeporte.rediris.es/revista/revista80/artefectos1197.htm

DOI: https://doi.org/10.15366/rimcafd2020.80.010

\title{
ORIGINAL
}

\section{EFECTOS DEL EJERCICIO SOBRE LA FLEXIBILIDAD EN PERSONAS MAYORES DE 65 AÑOS}

\section{EFFECTS OF EXERCISE ON FLEXIBILITY IN ADULTS OVER 65 YEARS OLD}

\author{
Matos-Duarte, M. ${ }^{1}$ y Berlanga, L.A. ${ }^{2}$ \\ ${ }^{1}$ Doctora en Ciencias de la Actividad Física y del Deporte. Profesora de la Facultad de Educación \\ de la Universidad Francisco de Vitoria (UFV) (España) michelle.matos@ufv.es \\ ${ }^{2}$ Profesor de la Facultad de Ciencias de la Salud de la Universidad Francisco de Vitoria (UFV) \\ (España) luis.berlanga@ufv.es
}

Código UNESCO / UNESCO code: 3212 Salud Pública / Public Health Clasificación Consejo de Europa / Council of Europe classification: 17 Otros (Actividad Física y Salud) / Others (Physical Activity and Health)

Recibido 28 de noviembre de 2018 Received November, 28, 2018 Aceptado 25 de junio de 2019 Accepted June 25, 2019

\section{RESUMEN}

Es sabido que el envejecimiento provoca el deterioro de la condición física y sus componentes. Menores niveles de flexibilidad se asocian a una pérdida de la capacidad funcional, y la participación en programas de ejercicio físico supervisado podría mantener o mejorar los niveles de dicha cualidad física. En nuestro estudio, evaluamos la flexibilidad de 53 adultos mayores de 65 años $(M=73,74 \pm 4,54$ años) que participaban en un programa de ejercicio durante, al menos, 6 meses; utilizando los tests back scratch (TBS) y chair sit and reach (TCSAR). Al finalizar el programa, los resultados de flexibilidad resultaron estar dentro de los valores normativos de referencia; estando, en muchas ocasiones, por encima. En conclusión, mantenerse físicamente activo minimiza la pérdida de flexibilidad inherente al proceso de envejecimiento, incluso cuando la actividad física no está directamente dirigida a mejorar esa capacidad. Este hecho podría tener importantes repercusiones sobre la capacidad funcional y la calidad de vida de los adultos mayores.

PALABRAS CLAVE: Flexibilidad, Test Back Scratch, Test Chair Sit And Reach, Actividad Física, Ejercicio, Personas Mayores 


\begin{abstract}
Aging involves physiological alterations such as detriments in physical fitness and its components. Lower levels of flexibility are associated to a loss of functional capacity, and participation in supervised physical exercise programs could maintain or improve levels of that physical ability. In our study, we analysed flexibility in 53 adults over 65 years old $(M=73,74 \pm 4,54)$ who participated in an exercise program for at least 6 months; using Back Scratch Test (TBS), and Chair Sit and Reach Test (TCSAR). At the end of the program, the results of flexibility were found to be within the normative values of reference; being, on many occasions exceeded them. In conclusion, staying physically active minimises the loss of flexibility inherent to the aging process, which could has an important impact on the functional capacity and quality of life of older adults.
\end{abstract}

KEY WORDS: Flexibility, Test Back Scratch, Test Chair Sit and Reach, Physical Activity, Exercise, Older Adults.

\title{
INTRODUCCIÓN
}

El envejecimiento es un proceso inherente al ser humano que supone cambios fisiológicos, psicológicos y sociales en su vida. Entre las alteraciones fisiológicas, cabe destacar el deterioro de los componentes de la condición física, y, en particular, de la flexibilidad. Esta capacidad física tiende a disminuir con los años (Marques et al., 2014; Stathokostas, McDonald, Little y Paterson, 2013; Vagetti et al., 2015; Vaquero-Cristóbal, González-Moro, Ros y Alacid, 2012), pudiendo alcanzar pérdidas en la amplitud de movimiento de hasta un $40 \%$ en determinadas articulaciones (American College of Sports Medicine - ACSM, 2009).

La pérdida significativa de la flexibilidad se considera un agravante en la salud y la calidad de vida de las personas mayores (Fabre et al., 2007), siendo fundamental su mantenimiento para realizar las actividades cotidianas de manera autónoma y eficaz (Gonçalves, Barata, Varejão y Dantas, 2011). Su conservación está asociada a la prevención de caídas (Guimarães y Farinatti, 2005), el desempeño adecuado de las actividades de la vida diaria (Brach y VanSwearingen, 2002; Geraldes, Albuquerque, Soares, Carvalho y Farinatti, 2008; Stanziano, Roos, Perry, Lai y Signorile, 2009), el mantenimiento de la locomoción (Cristopoliski, Sarraf, Dezan, Provensi y Rodacki, 2008; Schenatto, Milano, Berlezi y Bonamigo, 2009), la prevención de alteraciones posturales (ACSM, 2005; Da Silva Dias y Gómez-Conesa, 2008) y la disminución de dolores musculares (King et al., 2000; Ponce, Sempere y Cortés, 2014).

La flexibilidad es una capacidad física imprescindible para el correcto funcionamiento del organismo y la preservación de la salud, sin embargo, las 
investigaciones científicas en torno a esta capacidad física son aún insuficientes y ciertamente contradictorias, debido a la heterogeneidad metodológica y de las muestras analizadas (Fiatarone Singh, 2002; Rikli y Jones, 2013).

Aun así, parece haber un consenso generalizado sobre la importancia de mantener la extensibilidad de los músculos y las articulaciones ágiles y con buena movilidad para mantener una buena capacidad funcional. En las personas mayores esta es una cuestión importante, pues el proceso de envejecimiento interfiere en los niveles de flexibilidad debido tanto a las alteraciones biológicas propias de la edad avanzada, como al sedentarismo y la inactividad física que se ven más acentuados en esta fase de la vida (Guthold, Ono, Strong, Chatterji y Morabia, 2008), empeorando la amplitud de movimiento como consecuencia de la falta de uso de las articulaciones (Heyward, 2008).

Las recomendaciones actuales de actividad física sugieren que el aumento de la condición física con la práctica de ejercicios de flexibilidad combate la tendencia a la pérdida de amplitud de movimiento (Correa-Bautista, Sandoval-Cuellar, Alfonso-Mora y Rodríguez-Daza, 2012; Hulya, Sevi, Serap y Ayse, 2015; Toto et al., 2012; Vieira et al., 2015). En consecuencia, lo más coherente para la tercera edad es la adopción de un estilo de vida activo para beneficiarse de articulaciones suficientemente flexibles como para seguir realizando las acciones básicas de la vida diaria con autonomía e independencia. De esta manera, que las personas mayores incluyan en su día a día la reproducción de movimientos habituales ejecutados en su máxima amplitud podría asegurar un cierto grado de estiramiento (Andújar, 2010). Asimismo, sería recomendable su participación en programas estructurados de ejercicio físico que desarrollen la condición física global, incluyendo el trabajo específico de la flexibilidad.

Por tanto, el estilo de vida activo adoptado por cada persona será determinante no solo para preservar la amplitud de movimientos, sino también para retrasar la pérdida de funcionalidad con los años (Coelho de Farias, Borba-Pinheiro, Oliveira y Gomes de Souza 2014; Vaquero-Cristóbal, López-Miñarro, AlacidCárceles y Esparza-Ros, 2015). En consecuencia, favorecerá el buen funcionamiento del cuerpo consiguiendo que la persona experimente un envejecimiento más exitoso, ya que la flexibilidad está asociada a la calidad de vida del individuo mayor (Gonçalves et al., 2011; Sławińska, Posłuszny y Rożek, 2013).

Para determinar los niveles de flexibilidad existen diversos métodos, siendo los test lineales los que se consideran más fáciles de aplicar y de ser reproducidos por los sujetos, además de utilizar materiales simples y económicos en su medición (Geraldes, Cavalcante, Albuquerque, Carvalho y Farinatti, 2007). En las personas de edad avanzada las pruebas de flexibilidad de la batería Senior Fitness Test (SFT) desarrolladas por Rikli y Jones (2001) son altamente recomendadas, debido a su seguridad, la facilidad de ejecución de los movimientos y su buena confiabilidad y validez comprobada (Rikli y Jones, 1999a). Estas pruebas incluyen el Test Back Scratch (TBS), que mide la amplitud 
de movimiento del tren superior, especialmente de los hombros; y el Test Chair Sit and Reach (TCSAR), que evalúa la del tren inferior.

Al medir la flexibilidad con las pruebas TBS y TCSAR, los resultados se pueden comparar con los valores de referencia estándar, recopilados por las autoras del SFT (Rikli y Jones, 2001). Esta herramienta de evaluación es importante para que las personas mayores puedan conocer sus resultados y compararlos con los valores obtenidos por poblaciones del mismo género y rango de edad. Además, es un instrumento válido para que los profesionales del área de la salud y de la actividad física, puedan evaluar con facilidad y precisión el estado en el que se encuentra la movilidad articular de la población con edad avanzada. El conocimiento del estado de la salud articular, permitirá estructurar estrategias y programas de ejercicio físico enfocados específicamente al desarrollo y mejora de la capacidad funcional de estas personas.

En este contexto, el objetivo de este estudio es conocer el nivel de movilidad articular del tren superior e inferior en un grupo de personas mayores físicamente activas para compararlo con los valores de referencia internacionalmente establecidos.

\section{MATERIAL Y MÉTODOS}

\section{Muestra}

El presente estudio incluyó a 53 voluntarios brasileños (14 varones y 39 mujeres) mayores de 65 años ( $M=73,74 \pm 4,54$ años), todos ellos participantes del Programa Actividad Física y Salud en Personas Mayores (Programa Atividade Física e Saúde do Idoso, PAFSI) de la Universidad Federal de Minas Gerais (UFMG), ubicada en la ciudad de Belo Horizonte, Brasil. Para la selección de la muestra se establecieron como requisitos que los voluntarios tuvieran la edad mínima de 65 años y que estuvieran participando en el programa PAFSI durante, al menos, los seis meses anteriores al inicio del estudio. Asimismo, tomando como referencia la definición de autonomía e independencia sugerida por Moraes (2012), como criterio de inclusión se consideró que los sujetos de la muestra fueran funcionalmente independientes; y que contaran con un certificado médico de conformidad con la realización de actividad física y que no se apreciaran signos de padecer limitaciones musculoesqueléticas y cognitivas que pudieran comprometer la ejecución de los movimientos a ser evaluados.

\section{Programa de ejercicio físico administrado}

EI PAFSI fue administrado por profesionales graduados en Ciencias de la Actividad Física y del Deporte. Además de contar con un programa estructurado de ejercicio físico, el proyecto contaba con actividades sociales y de ocio fuera del espacio de la universidad y de los horarios habituales. Asimismo, el programa incluía seminarios y charlas informativas con la finalidad de orientar y concienciar 
a los participantes sobre temas de gran importancia para la salud y la calidad de vida en el envejecimiento, como la nutrición o la prevención de caídas.

El programa consistía en tres sesiones semanales de 60 minutos. En general, la estructura de las clases se dividía en un calentamiento inicial de unos 15 minutos, que incluía caminata, ejercicios de movilidad articular y estiramientos estáticos. A continuación, se realizaba la parte principal de la sesión de unos $35 \mathrm{mi}-$ nutos, en la cual se trabajaban los diversos aspectos de la condición física global a través de actividades globales variadas (juegos, bailes, deportes adaptados y ejercicios cardiovasculares, de fuerza, de equilibrio, de coordinación y de flexibilidad). Por último, las sesiones finalizaban con ejercicios de relajación, respiración y estiramientos para la vuelta a la calma, durante unos 10 minutos.

\section{Instrumentos}

Para la evaluación de la flexibilidad se utilizaron las pruebas TBS y el TCSAR propuestos por Rikli y Jones (2001), que son pruebas con buena confiabilidad ( $r$ $=0,96$ y $r=0,95$, respectivamente) y validez comprobada (Rikli y Jones, 1999a), siendo además seguras y fáciles de llevar a cabo. Estas autoras realizaron un estudio en los Estados Unidos con una muestra de 7.183 sujetos de entre 60 y 94 años de edad, estableciendo valores de referencia tanto para la flexibilidad, como para el resto de las capacidades físicas (Rikli y Jones, 1999b), diferenciando entre géneros y franjas de edad de cinco años.

Para la realización de estas pruebas se respetaron todos los protocolos establecidos por Rikli y Jones (2001), en lo que se refiere al calentamiento previo, proporcionar la información pertinente a los participantes sobre la ejecución de los movimientos, y la reproducción de los mismos por parte de los evaluadores para ejemplificar; así como un breve período de tiempo para que los participantes pudieran familiarizarse con los movimientos y elegir el lado de preferencia a ser medido. También se siguieron los procedimientos estándar específicos en la aplicación de cada prueba, su puntuación, registro, análisis de los datos y las normas de seguridad para la ejecución.

Los test fueron administrados por un solo evaluador con conocimiento sobre los protocolos de cada prueba y con experiencia en la aplicación de los mismos. La toma de datos se extendió durante dos días, con la recolección de las medidas siendo realizadas siempre por la mañana, en la propia universidad y utilizando una sala previamente acondicionada para el desarrollo óptimo de las valoraciones.

Para contrastar los datos de la muestra con los valores de referencia internacional, se utilizaron las tablas de flexibilidad del SFT con sus valores normativos transformados en centímetros, como se representa en las tablas 1 y 2 : 
Tabla 1. Valores normativos de referencia para hombres

\begin{tabular}{cccccccc}
\hline Edad & $\mathbf{6 0 - 6 4}$ & $\mathbf{6 5 - 6 9}$ & $\mathbf{7 0 - 7 4}$ & $\mathbf{7 5 - 7 9}$ & $\mathbf{8 0 - 8 4}$ & $\mathbf{8 5 - 8 9}$ & $\mathbf{9 0 - 9 4}$ \\
\hline $\begin{array}{c}\text { Test } \\
\text { chair sit }\end{array}$ & $-6,4 \mathrm{a}$ & $-7,6 \mathrm{a}$ & $-7,6 \mathrm{a}$ & $-10,2 \mathrm{a}$ & $-14,0 \mathrm{a}$ & $-14,0 \mathrm{a}$ & $-16,5 \mathrm{a}$ \\
and reach & $+10,2$ & $+7,6$ & $+7,6$ & $+5,1$ & $+3,8$ & $+1,3$ & $-1,3$ \\
& & & & & & & \\
$\begin{array}{c}\text { Test } \\
\text { back scra- } \\
\text { tch }\end{array}$ & $-16,5 \mathrm{a}$ & $-19,1 \mathrm{a}$ & $-20,3 \mathrm{a}$ & $-22,9 \mathrm{a}$ & $-24,1 \mathrm{a}$ & $-24,1 \mathrm{a}$ & $-26,7 \mathrm{a}$ \\
& $+0,0$ & $-2,5$ & $-2,5$ & $-5,1$ & $-5,1$ & $-7,6$ & $-10,2$ \\
\hline
\end{tabular}

Nota: Valores transformados en centímetros.

Fuente: Adaptado de Rikli, R. E. y Jones, C. J. (2001). Senior Fitness Test Manual. Champaign, IL: Human Kinetics. p.143.

Tabla 2. Valores normativos de referencia para mujeres

\begin{tabular}{cccccccc}
\hline Edad & $\mathbf{6 0 - 6 4}$ & $\mathbf{6 5 - 6 9}$ & $\mathbf{7 0 - 7 4}$ & $\mathbf{7 5 - 7 9}$ & $\mathbf{8 0 - 8 4}$ & $\mathbf{8 5 - 8 9}$ & $\mathbf{9 0 - 9 4}$ \\
\hline $\begin{array}{c}\text { Test } \\
\text { chair sit }\end{array}$ & $-1,3 \mathrm{a}$ & $-1,3 \mathrm{a}$ & $-2,5 \mathrm{a}$ & $-3,8 \mathrm{a}$ & $-5,1 \mathrm{a}$ & $-6,4 \mathrm{a}$ & $-11,4 \mathrm{a}$ \\
and reach & $+12,8$ & $+11,4$ & $+10,2$ & $+8,9$ & $+7,6$ & $+6,4$ & $+2,5$ \\
& & & & & & & \\
$\begin{array}{c}\text { Test } \\
\text { back scra- } \\
\text { tch }\end{array}$ & $-7,6 \mathrm{a}$ & $-8,9 \mathrm{a}$ & $-10,2 \mathrm{a}$ & $-12,8 \mathrm{a}$ & $-14,0 \mathrm{a}$ & $-17,8 \mathrm{a}$ & $-20,3 \mathrm{a}$ \\
& $+3,8$ & $+3,8$ & $+2,5$ & $+1,3$ & $+0,0$ & $-2,5$ & $-2,5$ \\
\hline
\end{tabular}

Nota: Valores transformados en centímetros.

Fuente: Adaptado de Rikli, R. E. y Jones, C. J. (2001). Senior Fitness Test Manual. Champaign, IL: Human Kinetics. p.143.

Para la realización del análisis estadístico se utilizó el programa estadístico Statistical Package for the Social Sciences - SPSS para Windows en la versión 22.0. Se realizó un análisis descriptivo de las variables de la edad de la muestra, además de las variables investigadas conteniendo los valores medios, máximos, mínimos y desviaciones estándar. Se aplicaron la prueba de normalidad (Kolmogorov-Smirnov) para las variables y la prueba de Levene para verificar la homogeneidad de las varianzas. El nivel de significancia adoptado fue $p<0,05$.

Toda la información pertinente para el estudio se proporcionó a los participantes, que firmaron el correspondiente consentimiento informado. La identidad de la muestra fue preservada en anonimato, teniendo los datos recogidos el único fin de la investigación. Se atendieron las consideraciones que establece el Comité de Ética de Investigación de la Universidad Autónoma de Madrid, en asociación con el Departamento de Educación Física, Deporte y Motricidad Humana y el Departamento de Postgrado en Ciencias del Deporte de la UFMG. Como en todos los estudios que involucran seres humanos, se han respetado las normas éticas promulgadas por la Asociación Médica Mundial en la Declaración de Helsinki. 


\section{RESULTADOS}

Los resultados se presentan en tablas descriptivas, con valores de flexibilidad de la muestra separados por grupos de edad y género. Las pruebas estadísticas mostraron diferencias significativas entre hombres y mujeres en el grupo de 70 a 74 años para la prueba TBS, no observándose diferencias significativas en los demás grupos ni en los resultados del TCSAR.

Tabla 3. Análisis descriptivo de la flexibilidad del tren superior a través del TBS y del tren inferior a través del TCSAR en hombres y mujeres divididos por grupos de edad.

\begin{tabular}{|c|c|c|c|c|c|c|}
\hline \multirow[b]{2}{*}{ Edad } & \multirow[b]{2}{*}{$\mathbf{N}$} & \multicolumn{2}{|c|}{ Hombres } & \multicolumn{3}{|c|}{ Mujeres } \\
\hline & & $\begin{array}{c}\text { TBS } \\
\text { Media ( } \pm S D)\end{array}$ & $\begin{array}{c}\text { TCSAR } \\
\text { Media ( } \pm S D)\end{array}$ & $\mathbf{N}$ & $\begin{array}{c}\text { TBS } \\
\text { Media ( } \pm S D)\end{array}$ & $\begin{array}{c}\text { TCSAR } \\
\text { Media ( } \pm S D)\end{array}$ \\
\hline $65-69$ & 1 & $1,5 \pm 0$ & $0,7 \pm 0$ & 7 & $2,29 \pm 3,48$ & $0,09 \pm 11,51$ \\
\hline $70-74$ & 6 & $-10,50 \pm 2,57^{*}$ & $-5,35 \pm 5,6$ & 20 & $-3,44 \pm 8,98^{*}$ & $-2,08 \pm 7,74$ \\
\hline $75-79$ & 4 & $-12,15 \pm 10,41$ & $-11,02 \pm 13,68$ & 9 & $-3,08 \pm 13,73$ & $-2,52 \pm 12,15$ \\
\hline $80-84$ & 3 & $-5,93 \pm 2,80$ & $-12,37 \pm 10,95$ & 3 & $-14,6 \pm 5,6$ & $4,3 \pm 5,86$ \\
\hline
\end{tabular}

SD - desviación estándar; * $p<0,05$ en relación al test (diferencia entre hombres y mujeres)

La tabla 4 presenta los porcentajes de la muestra que se encontraron dentro, por encima y por debajo de los valores normativos de referencia según la clasificación propuesta por Rikli y Jones (2001). Los hombres presentaron en su mayoría valores iguales o superiores a los de referencia internacional en todos los grupos de edad tanto para el TBS como para el TCSAR, excepto en el grupo 75-79 años, en el cual un $50 \%$ estuvo por debajo del valor de referencia y un $50 \%$ por encima o dentro de estos valores normativos. La mayor parte de las mujeres tuvieron valores de flexibilidad dentro o superiores a los valores normativos de referencia en TBS y TCSAR, excepto en el grupo 80-84 años en TBS (66,7\% por debajo) y 70-74 años en TCSAR (50\% debajo).

Tabla 4. Porcentajes de la muestra en relación con los valores de referencia para hombres y mujeres en ambas pruebas.

\begin{tabular}{ccccccccccccc}
\hline & & \multicolumn{4}{c}{ Hombres } & & \multicolumn{4}{c}{ TCSAR } & \multicolumn{4}{c}{ TBS } & \multicolumn{3}{c}{ TCSAR } \\
Edad & Ref. & $\downarrow$ & $\uparrow$ & Ref. & $\downarrow$ & $\uparrow$ & Ref. & $\downarrow$ & $\uparrow$ & Ref. & $\downarrow$ & $\uparrow$ \\
\hline $\mathbf{6 5 - 6 9}$ & 0 & 0 & $100 \%$ & $100 \%$ & 0 & 0 & $57,1 \%$ & $14,3 \%$ & $28,6 \%$ & $42,9 \%$ & $42,9 \%$ & $14,2 \%$ \\
$\mathbf{7 0 - 7 4}$ & $100 \%$ & 0 & $0 \%$ & $83,3 \%$ & $16,7 \%$ & 0 & $60 \%$ & $15 \%$ & $25 \%$ & $45 \%$ & $50 \%$ & $5 \%$ \\
$\mathbf{7 5 - 7 9}$ & $50 \%$ & $25 \%$ & $25 \%$ & $25 \%$ & $50 \%$ & $25 \%$ & $33,3 \%$ & $22,2 \%$ & $44,5 \%$ & $33,3 \%$ & $44,5 \%$ & $22,2 \%$ \\
$\mathbf{8 0 - 8 4}$ & $66,7 \%$ & $33,3 \%$ & $0 \%$ & $66,7 \%$ & 0 & $33,3 \%$ & $33,3 \%$ & $66,7 \%$ & $0 \%$ & $66,7 \%$ & $33,3 \%$ & 0 \\
\hline
\end{tabular}

Ref. - dentro de los valores de referencia; “ $\downarrow$ ” - por debajo de los valores de referencia; “ por encima de los valores de referencia

\section{DISCUSIÓN}

Sabiendo que el envejecimiento es un proceso inherente en todas las personas y que supone la disminución de las capacidades fisiológicas, psicológicas y sociales del individuo; el primer hallazgo a resaltar de nuestro estudio es que el ejercicio físico supervisado consigue, al menos, mantener los niveles de 
flexibilidad en una población mayor de 65 años dentro de los valores normativos de referencia; consiguiendo, en muchos casos, que estos valores estén por encima de los normativos. Solo los sujetos de entre 75-79 años para el TCSAR, y las mujeres de entre 80-84 años para el TBS, estuvieron en su mayoría por debajo de los valores de referencia.

Estos resultados están en concordancia con los hallazgos de otros investigadores. Gonçalves et al. (2011) registraron una mejora de la flexibilidad medida con goniómetro en una muestra de 120 sujetos de 60 años o mayores, que realizaron dos sesiones de yoga a la semana de 60 minutos durante 14 semanas. Además, estos autores evaluaron pruebas funcionales relacionadas con las actividades de la vida diaria, donde la flexibilidad y la amplitud de movimiento son fundamentales para su ejecución, encontrando que el yoga mejoraba todas las pruebas excepto la de ponerse y quitarse una camiseta; concluyendo que la práctica regular de esta modalidad de ejercicio mejora la amplitud de movimiento y el desempeño de las tareas cotidianas, demostrando además mejoras importantes en la calidad de vida de los sujetos.

En la misma línea, Matos-Duarte, Martínez-de-Haro, Sanz-Arribas, Andrade y Chagas (2017) hallaron en una muestra de más de 65 años que la flexibilidad tanto del tren superior como del tren inferior, evaluada utilizando los mismos instrumentos que en nuestro estudio (TBS y TCSAR), sigue mejorando a lo largo del tiempo siempre y cuando los sujetos sigan activos en programas de ejercicio físico global que potencien el desarrollo de esta cualidad física.

En un estudio observacional donde participaron 74 personas mayores, Fabre et al. (2007) concluyeron que la pérdida de flexibilidad asociada al envejecimiento continua incluso al llegar a los 90 años, y supone una merma importante de la calidad de vida de estas personas. De hecho, la pérdida de flexibilidad asociada al envejecimiento y su relación con la capacidad funcional y la calidad de vida fue claramente definida por Sayer et al. (2008), en cuya revisión hablaban de un umbral de discapacidad relacionado con la pérdida de masa y fuerza muscular y de la capacidad de movimiento. Marmolejo, Medhanie y Tarleton (2018) afirman que las personas que tienen mayores niveles de flexibilidad presentan un mejor equilibrio muscular y postura corporal, lo cual se podría traducir en una mayor productividad y eficiencia muscular que disminuiría el riesgo de lesión potencialmente.

En cuanto a la diferencia de género observada en el TBS en la franja de edad de los 70 a los 74 años, Santos et al. (2012) también observaron diferencias significativas entre géneros a favor de las mujeres, habiendo evaluado la flexibilidad de 312 sujetos de 65 a 103 años con el empleo tanto del TBS como del TCSAR. Sin embargo, estos autores no presentaron un análisis detallado por franjas de edad, tal y como se clasifican los valores normativos de referencia, por lo que no podemos conocer dónde se presentó esta diferencia significativa ni si la respuesta al programa de ejercicio físico fue distinta en función de la edad de los participantes. 
Gouveia et al. (2013), en su muestra de 802 portugueses y portuguesas de entre 60 y 79 años, encontraron que las mujeres obtuvieron puntuaciones significativamente mejores que los hombres en el TSCAR y el TBS, aunque los hombres presentaran mejor condición física general que las mujeres. Algo importante que concluyeron estos autores es que encontraron mayores mejoras de las variables evaluadas en aquellos sujetos con mayor participación en programas de actividad y ejercicio físico, con los importantes beneficios sobre la capacidad funcional y la calidad de vida de los sujetos que esto supone.

Por tanto, analizar la evolución de los niveles de flexibilidad en personas mayores es de vital importancia por su implicación en el desarrollo de actividades cotidianas y para ayudarles a preservar su autonomía, previniendo la discapacidad funcional que afecta a gran parte de la población mayor y a su calidad de vida. Sin embargo, una limitación de nuestro estudio fue que no incluimos el registro de esta variable. Además, los valores normativos de referencia de Rikli y Jones (1999b) se validaron para una muestra estadounidense; por lo que sería recomendable validar unos valores de referencia específicos para diferentes países.

\section{CONCLUSIÓN}

Concluimos con nuestro estudio que, según los parámetros internacionalmente establecidos, nuestra muestra de personas mayores de 65 años que participan en un programa de ejercicio físico supervisado, puede mantener niveles de movilidad articular y amplitud de movimiento adecuados. Sin embargo, se recomienda aumentar la carga de trabajo específicamente orientada al desarrollo de estas cualidades físicas en las personas mayores, con el fin de mejorar la autonomía y la capacidad funcional de dicha población.

\section{REFERENCIAS BIBLIOGRÁFICAS}

American College of Sports Medicine (2005). Manual ACSM para la valoración y prescripción del ejercicio ( $2^{a}$ ed.). Badalona: Paidotribo.

American College of Sports Medicine (2009). ACSM Position stand: Exercise and physical activity for older adults. Medicine \& Science in Sports \& Exercise, 41(7), 1510-1530. doi: 10.1249/MSS.0b013e3181a0c95c

Andújar, A. J. C. (2010). El desarrollo de la condición física para la mejora de la salud en adultos y personas mayores. En V. Martínez de Haro (Comp.), Actividad física, salud y calidad de vida (pp. 151 -178). Madrid: Fundación Estudiantes, Universidad Autónoma de Madrid y autores.

Brach, J. S. y VanSwearingen, J. M. (2002). Physical impairment and disability: Relationship to performance of activities of daily living in communitydwelling older men. Physical Therapy, 82(8), 752-761. doi: https://doi.org/10.1093/ptj/82.8.752

Coelho de Farias, M., Borba-Pinheiro, C., Oliveira, M. y Gomes de Souza, R. (2014). Efectos de un programa de entrenamiento concurrente sobre la 
fuerza muscular, flexibilidad y autonomía funcional de mujeres mayores. Revista Ciencias de la Actividad Física UCM, 15(2), 13-24.

Correa-Bautista, J. E., Sandoval-Cuellar, C., Alfonso- Mora, M. L. y RodríguezDaza, K. D. (2012). Cambios en la aptitud física en un grupo de mujeres adultas mayores bajo el modelo de envejecimiento activo. Revista de la Facultad de Medicina, 60(1), 21-30.

Cristopoliski, F., Sarraf, T. A., Dezan, V. H., Provensi, C. L. y Rodacki, A. L. (2008). Efeito transiente de exercícios de flexibilidade na articulação do quadril sobre a marcha de idosas. Revista Brasileira de Medicina do Esporte, 14(2), 139-144. https://doi.org/10.1590/S1517-86922008000200011

Da Silva Dias, R. y Gómez-Conesa, A. (2008). Síndrome de los isquiotibiales acortados. Fisioterapia, 30(4), 186-193. doi: 10.1016/j.ft.2008.07.004

Fabre, J. M., Wood, R. H., Cherry, K. E., Su, L. J., Cress, M. E., King, C. M., ... Jazwinski, S. M. (2007). Age-related deterioration in flexibility is associated with health-related quality of life in nonagenarians. Journal of Geriatric Physical Therapy, 30(1), 16-22. doi: 10.1519/00139143-20070400000004

Fiatarone Singh, M.A. (2002). Exercise comes of age: rationale and recommendations for a geriatric exercise prescription. Journals of Gerontology. Medical Sciences, 57A(5), M262-M282. doi.org/10.1093/gerona/57.5.M262

Geraldes, A. A., Albuquerque, R., Soares, R. M., Carvalho, J. y Farinatti, P. T.V. (2008). Correlação entre flexibilidade das articulações glenoumerais e coxofemorais e o desempenho funcional de idosas fisicamente ativas. Revista Brasileira de Fisioterapia, 12(4), 274-282. doi: https://doi.org/10.1590/S1413-35552008000400005

Geraldes, A. A., Cavalcante, A. P., Albuquerque, R., Carvalho, M. J. y Farinatti, P. T. V. (2007). Correlação entre a flexibilidade multiarticular e o desempenho funcional de idosas fisicamente ativas em tarefas motoras selecionadas. Revista Brasileira de Cineantropometria \& Desempenho Humano, 9(3), 238-243.

Gonçalves, L., Vale, R., Barata, N. J., Varejão, R. V. y Dantas, E. H. (2011). Flexibility, functional autonomy and quality of life (QoL) in elderly yoga practitioners. Archives of Gerontology and Geriatrics, 53(2), 158-162. doi: 10.1016/j.archger.2010.10.028

Gouveia, E. R., Maia, J. A., Beunen, G. P., Blimkie, C. J., Fena, E. M. y Freitas, D. L. (2013). Functional fitness and physical activity of portuguese community-residing older adults. Journal of Aging and Physical Activity, 21(1), 1-19. doi: https://doi.org/10.1123/japa.21.1.1

Guimarães, J. M. N. y Farinatti, P. T. V. (2005). Análise descritiva de variáveis teoricamente associadas ao risco de quedas em mulheres idosas. Revista Brasileira de Medicina do Esporte, 11(5), 299-305. doi: https://doi.org/10.1590/S1517-86922005000500011

Guthold, R., Ono, T., Strong, K. L., Chatterji, S. y Morabia, A. (2008). Worldwide variability in physical inactivity a 51-country survey. American Journal of Preventive Medicine, 34(6), 486-494. doi: 10.1016/j.amepre.2008.02.013 
Heyward, V. H. (2008). Evaluación de la aptitud física y prescripción del ejercicio (5⿳⺈冂䒑 ed.). Madrid: Panamericana.

Hulya, T. D., Sevi, Y., Serap, A. y Ayse, O. (2015). Factors affecting the benefits of a six-month supervised exercise program on community-dwelling older adults: Interactions among age, gender, and participation. Journal of Physical Therapy Science, 27(5), 1421-1427. doi: 10.1589/jpts.27.1421

King, A. C., Pruitt, L. A., Phillips, W., Oka, R., Rodenburg, A. y Haskell, W. L. (2000). Comparative effects of two physical activity programs on measured and perceived physical functioning and other health-related quality of life outcomes in older adults. Journals of Gerontology Series A: Biological Sciences and Medical Sciences, 55(2), M74-M83. doi:10.1093/gerona/55.2.M74

Marmolejo, M. A., Medhanie, M. y Tarleton, H. P. (2018). Musculoskeletal flexibility and quality of life: A Feasibility study of homeless young adults in Los Angeles County. International Journal of Exercise Science, 11(4), 968-979.

Marques, E. A., Santos, D. A., Silva, A. M., Baptista, F., Santos, R., Vale, S., ...Sardinha, L. B. (2014). Normative functional fitness standards and trends of portuguese older adults: Cross-cultural comparisons. Journal of Aging and Physical Activity, 22(1), 126-137. doi: 10.1123/JAPA:2012-0203

Matos-Duarte, M.; Martínez-de-Haro, V.; Sanz-Arribas, I.; Andrade, A.G.P. y Chagas, M.H. (2017). Estudio longitudinal de la flexibilidad funcional en mayores físicamente activos. Revista Internacional de Medicina $y$ Ciencias de la Actividad Física y el Deporte, 17(65), 121-137. doi: http://dx.doi.org/10.15366/rimcafd2017.65.008

Moraes, E. N. (2012). Atenção à saúde do idoso: Aspectos conceituais. Brasília: Organização Pan-Americana da Saúde.

Ponce, M. J., Sempere, N. y Cortés, S. (2014). Efectividad de un programa de ejercicios dise-ado para personas con osteoporosis y osteopenia en el manejo del dolor y la calidad de vida. European Journal of Investigation in Health, Psychology and Education, 4(3), 169-179. doi: 10.1989/ejihpe.v4i3.64

Rikli, R. E. y Jones, C. J. (1999a). Development and validation of a functional fitness test for community-residing older adults. Journal of Aging and Physical Activity, 7, 129-161. doi: https://doi.org/10.1123/japa.7.2.129

Rikli, R. E. y Jones, C. J. (1999b). Functional fitness normative scores for communityresiding older adults, ages 60-94. Journal of Aging and Physical Activity, 7, 162-181. doi: https://doi.org/10.1123/japa.7.2.162

Rikli, R. E. y Jones, C. J. (2001). Senior Fitness Test Manual. Champaign, IL: Human Kinetics.

Rikli, R. E. y Jones, C. J. (2013). Development and validation of criterionreferenced clinically relevant fitness standards for maintaining physical independence in later years. Gerontologist, 53(2), 255-267. doi: 10.1093/geront/gns071

Santos, D. A., Silva, A. M., Baptista, F., Santos, R., Vale, S., Mota, J. y Sardinha, L. B. (2012). Sedentary behavior and physical activity are independently 
related to functional fitness in older adults. Experimental Gerontology, 47(12), 908-912. doi: 10.1016/j.exger.2012.07.011

Sayer, A. A., Syddall, H., Martin, H., Patel, H., Baylis, D. y Cooper, C. (2008). The developmental origins of sarcopenia. Journal of Nutrition Health and Aging, 12(7), 427-432. doi: 10.1007/BF02982703

Schenatto, P., Milano, D., Berlezi, E. y Bonamigo, E. (2009). Relação entre aptidão muscular e amplitude articular, por faixa etária, na marcha do idoso. Revista Brasileira de Geriatria e Gerontologia, 12(3), 377-389. https://doi.org/10.1590/1809-9823.2009.00006

Sławińska, T., Posłuszny, P. y Rożek, K. (2013). The relationship between physical fitness and quality of life in adults and the elderly. Human Movement, 14(3), 200-204. doi: 10.2478/humo-2013-0023

Stanziano, D. C., Roos, B. A., Perry, A. C., Lai, S. y Signorile, J. F. (2009). The effects of an active-assisted stretching program on functional performance in elderly persons: A pilot study. Clinical Interventions in Aging, 4, 115-120. doi: $10.2147 /$ cia.s4152

Stathokostas, L., McDonald, M. W., Little, R. M. y Paterson, D. H. (2013). Flexibility of older adults aged 55-86 years and the influence of physical activity. Journal of Aging Research, 2013, 1-8. doi: 10.1155/2013/743843

Toto, P. E., Raina, K. D., Holm, M. B., Schlenk, E. A., Rubinstein, E. N. y Rogers, J. C. (2012). Outcomes of a multicomponent physical activity program for sedentary, community-dwelling older adults. Journal of Aging and Physical Activity, 20(3), 363-378. https://doi.org/10.1123/japa.20.3.363

Vagetti, G. C., Barbosa Filho, V., Oliveira, V., Mazzardo, O., Moreira, N., Gomes, A. C. y Campos, W. (2015). Functional fitness in older women from southern Brazil: Normative scores and comparison with different countries. Revista Brasileira de Cineantropometria \& Desempenho Humano, 17(4), 472-484. doi: 10.5007/1980-0037.2015v17n4p472

Vaquero-Cristóbal, R., González-Moro, I., Ros, E. y Alacid, F. (2012). Evolución de la fuerza, flexibilidad, equilibrio, resistencia y agilidad de mujeres mayores activas en relación con la edad. Motricidad. European Journal of Human Movement, 29, 29-47.

Vaquero-Cristóbal, R., López-Miñarro, P., Alacid-Cárceles, F. y Esparza-Ros, F. (2015). Efectos del método Pilates sobre la extensibilidad isquiosural, la inclinación pélvica y la flexión del tronco. Nutrición Hospitalaria, 32(5), 1967-1986. doi: 10.3305/nh.2015.32.5.9678

Vieira, E., Gurgel, J., Maia, T., Porto, F., Louro, J., Silva, E. y Alves Junior, E. (2015). Reach capacity in older women submitted to flexibility training. Revista Brasileira de Cineantropometria \& Desempenho Humano, 17(6), 722-732. doi: 10.5007/1980-0037.2015v17n6p722

\section{Número de citas totales / Total references: 39 (100\%) Número de citas propias de la revista / Journal's own references: 1 (2,6\%)}

Rev.int.med.cienc.act.fís.deporte - vol. 20 - número 80 - ISSN: 1577-0354 